\title{
MEK1/2 Inhibitors Induce Class 1 Alcoholic Dehydrogenase (ADH1) Expression by Regulating Farnesoid X Receptor
}

\author{
Xiaolin Wang \\ Nankai University \\ Yanfang Yang \\ Nankai University \\ Yuanli Chen \\ Hefei University of Technology \\ Yajun Duan \\ Hefei University of Technology \\ Jihong Han \\ Nankai University \\ Xiaoxiao Yang ( $\nabla$ yangxiaoxiao@hfut.edu.cn ) \\ Hefei University of Technology https://orcid.org/0000-0003-0290-2844
}

\section{Research Article}

Keywords: Alcohol, ADH1, ERK1/2, MEK1/2 inhibitor, FXR

Posted Date: October 25th, 2021

DOI: https://doi.org/10.21203/rs.3.rs-981887/v1

License: (c) (i) This work is licensed under a Creative Commons Attribution 4.0 International License. Read Full License 


\section{Abstract}

Background: Alcohol is mainly catabolized by class 1 alcohol dehydrogenase (ADH1) in liver. ADH deficiency can aggravate ethanol-induced tissue injury. Extracellular signal-regulated kinases $1 / 2$ (ERK1/2) is involved in alcohol metabolism. However, the relationship between ERK1/2 and ADH1 remains unclear.

Methods and Results: To inhibit ERK1/2, HepG2 and BNL cells were treated with mitogen-activated protein kinases 1/2 (MEK1/2) inhibitors (U0126 and PD98059), and C57BL/6J mice were fed U0126. After treatment, the protein and mRNA expression of $A D H 1$ were determined by Western blot and quantitative real time-PCR. The activity of ADH1 promoter was detected using luciferase assay. The results showed MEK1/2 inhibitors significantly increased ADH1 protein expression by inducing its transcription activity. Then we found a putative farnesoid $X$ receptor (FXR) response element (FXRE) sequence in $\mathrm{ADH} 1$ promoter by completing a sequence alignment analysis. To test whether FXR mediates the induction of MEK1/2 inhibitors on ADH1, HepG2 cells were transfected with FXR siRNA or ADH1 promoters with FXRE mutation. We found both FXR siRNA and FXRE mutation in ADH1 promoter abolished MEK1/2 inhibitors-induced ADH1 expression, indicating the activation of MEK1/2 inhibitors on ADH1 depends on FXR.

Conclusions: Our findings revealed inhibition of ERK1/2 can significantly increase ADH1 expression, indicating MEK1/2 inhibitors may possess potential application in alcohol-related diseases.

\section{Introduction}

Alcohol consumption plays a traditional role in many countries. However, numerous studies have demonstrated alcohol is harmful to human body. It can cause cognitive deficits, such as difficulties in problem-solving, memory and learning (1). Alcohol also induces dysfunction of immune system and increases the risk of cancers $(2,3)$. Thus, to avoid alcohol-induced toxicity, accelerating alcohol metabolism is quite important. It is reported that only $2-10 \%$ of alcohol in body is excreted through the lungs, urine and sweat, while most of the remainder is catabolized in liver (4). These data indicate liver bears a heavy burden of alcohol metabolism. Correspondingly, alcohol pose a great threat to liver. About $48 \%$ of liver cirrhosis deaths was attributed to alcohol. The mortality from liver cancer secondary to alcohol for 2010 was estimated to be 149,000 deaths (5).

Alcohol dehydrogenase (ADH), a zinc-containing enzyme, is highly expressed in liver. It is the major pathway for alcohol metabolism (6). $75 \%-80 \%$ of alcohol in liver proceeds via ADH (5). ADH catalyzes ethanol into acetaldehyde which is then quickly converted into acetate by aldehyde dehydrogenase. There are several forms of ADH, such as class 1 ADH (ADH1), class 2 AHD (ADH4) and class 3 ADH (ADH5). Among these forms, ADH1 plays the most important role in alcohol metabolism (6). Therefore, induction of $A D H 1$ is beneficial to alcohol elimination. 
Extracellular signal-regulated kinases 1 and 2 (ERK1/2) are involved in a variety of biological processes, including cell survival, metabolism and transcription (7). Mitogen-activated protein kinases 1/2 (MEK1/2) is required to phosphorylate and activate ERK1/2. Several studies have demonstrated that phosphorylation of ERK1/2 in liver is increased after alcohol consumption in rats and mice $(8,9)$. ERK1/2 has also been reported to be associated with ethanol-induced liver injury (10). These papers suggest ERK1/2 activation may hinder ethanol metabolism. Therefore, we hypothesis that inhibition of ERK1/2 by MEK1/2 inhibitors can active ADH1 expression in hepatocytes, thereby increasing ethanol metabolism.

\section{Materials And Methods}

\section{Reagents}

U0126 (Cat\# U6770) and PD98059 (Cat\# P4313) were purchased from LC laboratories (Woburn, MA, USA). Mouse anti-ADH (Cat\# sc-137078) monoclonal antibody was purchased from Santa Cruz Biotechnology (Dallas, Texas, USA). Rabbit anti-FXR (Cat\# NBP2-16550) polyclonal antibody was purchased from Novus Biologicals (Littleton, CO, USA). HRP-conjugated anti-GAPDH (Cat\# HRP-60004), HRP-conjugated goat anti-mouse IgG (H+L, Cat\# A00001-1) and rabbit IgG (Cat\# A00001-2) antibodies were purchased from Proteintech Group (Chicago, IL, USA). Rabbit anti-phosphorylated ERK1/2 (piERK1/2, Cat\# 9101) and ERK1/2 (Cat\# 9102) polyclonal antibodies were purchased from Cell Signaling Technology (Beverly, MA, USA). Human FXR siRNA (Cat\# sc-38848) was purchased from Santa Cruz Biotechnology (Dallas, Texas, USA).

\section{In vivo study}

The protocol for in vivo study was granted by the Ethics Committee of Nankai University and conforms to the Guide for the Care and Use of Laboratory Animals published by NIH. The mice were housed in SPF units of the Animal Center at Nankai University (with a 12-h light cycle from 8 a.m. to 8 p.m., $23 \pm 1^{\circ} \mathrm{C}, 60$ $70 \%$ humidity) with free access to normal chow and water. Up to five mice were kept per ventilated cage with corn cob bedding material. Male C57BL/6J mice ( 6-week old, $\sim 22 \mathrm{~g}$ ) were randomly divided into two groups ( 5 mice/group) and fed normal chow (control group) or normal chow containing U0126 (1.5 $\mathrm{mg} /$ day $/ \mathrm{kg}$ bodyweight, U0126 group) for five weeks. At the end of experiment, all mice were anesthetized and euthanized in a $\mathrm{CO}_{2}$ chamber followed by collection of liver samples.

\section{Cell culture}

HepG2 (a human hepatic cell line) and BNL (a murine hepatic cell line) cells were purchased from ATCC (Rockville, MD, USA) and cultured in DMEM medium containing $10 \%$ fetal bovine serum, $50 \mathrm{mg} / \mathrm{mL}$ penicillin/streptomycin and $2 \mathrm{mM}$ glutamine. 


\section{Western blot}

Lysate was prepared from mouse liver or cell samples with the protein lysis buffer $(150 \mathrm{mM} \mathrm{NaCl}, 50 \mathrm{mM}$ Tris $\mathrm{pH}$ 7.5, $50 \mathrm{mM}$ sodium fluoride, $1 \%$ Triton $\mathrm{X}-100,1 \%$ sodium deoxycholate, $1 \mathrm{mM}$ PMSF, $1 \mathrm{mM}$ sodium orthovanadate, $50 \mathrm{mg} / \mathrm{mL}$ aprotinin/leupeptin), followed by quantification of protein concentration. Protein expression of pi-ERK1/2, ERK1/2, ADH1 and FXR were determined by Western blot (11).

\section{Quantitative real time-PCR (qRT-PCR)}

Total RNA was isolated from samples using TRIzol reagent. CDNA was synthesized with a reverse transcription kit. Gene expression was determined by qRT-PCR using SYBR Green PCR reagents and following primers: homo-ADH1A forward, 5'-ATCAACATGAGCACAGCAGG-3', and homo-ADH1A backward, 5'-ATCAACATGAGCACAGCAGG-3'; homo-ADH1B forward, 5'-TCCCAAAACTTGTGGCTGAT-3', and homoADH1B backward, 5'-ATTGCCTCAAAACGTCAGGA-3'; homo-ADH1C forward, 5'-

CAAGTACTCACCAGCCTCCTG-3', and homo-ADH1C backward, 5'-CTCCCATAGCACAGCTGCTT-3'; homoGAPGH forward, 5'-TGATGACATCAAGAAGGTGGTGAAG-3', and homo-GAPDH backward, 5'-

TCCTTGGAGGCCATGTGGGCCAT-3'; mus-ADH1 forward, 5'-CCACTGGACAAAGTCTGCCT-3', and musADH1 backward, 5'-ACAGACAGACCGACACCTCC-3'; mus-GAPGH forward, 5'-ACCCAGAAGACTGTGGATGG3', and mus-GAPDH backward, 5'-ACACATTGGGGGTAGGAACA-3'. mRNA levels were normalized by $G A P D H$ in the corresponding samples.

\section{Preparation of plasmid DNA and determination of ADH1 promoter activity}

Human ADH1A, ADH1B, ADH1C and mouse ADH1 promoters were constructed using PCR with genomic DNA extracted from HepG2 cells or mouse liver and following primers: homo-ADH1 A forward, 5'TGAACTCTTCTCCTGGCACG-3', and homo-ADH1A backward, 5'-TTGCTTACTTTTCCTGCTGTG-3'; homoADH1B forward, 5'-GGGATCGCTGACCCAAACT-3', and homo-ADH1B backward, 5'-

TTTTTGCTTACTTTTCCTGCTGT-3'; homo-ADH1C forward, 5'-TCGTACTATCCCTGATTGG-3', and homoADH1C backward, 5'-TTCTTCTCTGCTTGAGTGC-3'; homo-ADH1A-mut forward, 5'TGTATTGATTGCTCATCAGTGACCAACTTCTTTATGATTT-3', and homo-ADH1A-mut backward, 5'AAATCATAAAGAAGTTGGTCACTGATGAGCAATCAATACA-3'; homo-ADH1B-mut forward, 5'TATTTTGATTGCTCATCAGGGACCAGTTTATCTGTTTTTGA-3', and homo-ADH1B-mut backward, 5'TCAAAACAGATAAACTGGTCCCTGATGAGCAATCAAAATA-3'; homo-ADH1C-mut forward, 5'AGGTTTTGAGTGCTCATCAGGGACCAGTTTCTTTATGATTT-3', and homo-ADH1 C-mut backward, 5'AAATCATAAAGAAACTGGTCCCTGATGAGCACTCAAAACCT-3'; mus-ADH1 forward, 5'TCCCCTGAGGTGAGATGTGT-3', and mus-ADH1 backward, 5'-GACTCTGCATCTCTCCACGTA-3'. The PCR products were digested followed by ligation with $p G L 4$ luciferase reporter vector. To analyze promoter 
activity, cells in 24-well plates were transfected with plasmid for the ADH1 promoters or Renilla luciferase (for internal normalization) for $6 \mathrm{~h}$, then received indicated treatment. After treatment, cells were lysed and the cellular lysate was used to determine activities of Firefly and Renilla luciferases using the dualluciferase reporter assay system (Promega, Madison, USA).

\section{Data analysis}

All the experiments were repeated at least 3 times, and the representative results were presented. Values are presented as mean \pm SD. GraphPad Prism software (version 8) was used for one-way ANOVA with post hoc Tukey's test or unpaired t test (two-tailed) whenever appropriate. The significant difference was considered if $\mathrm{P}<0.05$.

\section{Results}

\section{MEK1/2 inhibitors induce ADH1 expression in human hepatic cells}

Alcohol is harmful to various tissues because of its toxic effects. Liver is a major organ for alcohol metabolism. Ethanol can promote phosphorylation of ERK $1 / 2$ and activated ERK $1 / 2$ is involved in alcohol-induce liver injury $(12,13)$. So, we hypothesized inhibition of ERK1/2 may protect hepatocytes against ethanol-induced cytotoxicity. MEK1/2 is the upstream kinase of ERK1/2. U0126 and PD98059 are two specific MEK1/2 inhibitors. Both of them can significantly reduce pi-ERK1/2 (the active form of EKR1/2) in HepG2 cells (Fig. 1a, b). Therefore, we used U0126 and PD98059 to inhibit ERK1/2 in this study. To determine our hypothesis, we treated HepG2 cells with ethanol, or ethanol plus U0126 and PD98059. As shown in Fig. $1 \mathrm{c}$ and d, ethanol greatly decreased cell survival rate [Fig. $1 \mathrm{c}: \mathrm{F}_{4,20}=8.06$, $P=0.009$ ], [Fig. 1d: $\left.F_{4,20}=5.397, P=0.0071\right]$. However, $U 0126$ and PD 98059 ameliorated ethanol-induced cell apoptosis, indicating inhibition of ERK1/2 can reduce ethanol toxicity [Fig. $1 \mathrm{c}$ : $F_{4,20}=8.07, P<0.001$ ], [Fig. 1d: $\left.F_{4,20}=5.4, P=0.019\right]$. $A D H$ is a rate-limiting enzyme in alcohol metabolism (4), so we speculated that U0126 and PD98059 may ameliorate ethanol-induced cytotoxicity by regulating ADH1 expression. As we expected, the results in Fig. 1e showed that $\mathrm{U} 0126$ induced ADH1 protein levels in a concentration dependent manner $\left[F_{2,6}=81.6, P<0.001\right]$. The time course study with $5 \mathrm{mM} \cup 0126$ demonstrated the induction occurred quickly ( $3 \mathrm{~h}$ after treatment) and lasted for $24 \mathrm{~h}$ of the treatment $\left[F_{5,12}=99, P<0.001\right]$ (Fig. 1f). Similarly, we found PD98059 also can increase $A D H 1$ protein expression $\left[F_{3,8}=121, P<0.001\right]$ (Fig. 1e).

Human ADH1 contains three genes, $A D H 1 A, A D H 1 B$ and $A D H 1 C$ which encode $\mathrm{a}, \mathrm{b}$, and $\mathrm{g}$ subunits respectively. These subunits can combine and function as homodimers or heterodimers (6). To determine if MEK1/2 inhibitors regulate $A D H 1$ protein expression at the transcriptional level, we detected mRNA expression of $A D H 1 A, A D H 1 B$ and $A D H 1 C$ in response to $\mathrm{U} 0126$ treatment. The results in both 
concentration and time course studies demonstrate that U0126 greatly increased $A D H 1 A, A D H 1 B$ and $A D H 1 C$ mRNA levels [Fig. 2a, $A D H 1 A: \mathrm{F}_{2,12}=166.2$, $\mathrm{P}<0.001$ ], [Fig. 2a, $A D H 1 B: \mathrm{F}_{2,12}=37, \mathrm{P}<0.001$ ], [Fig. 2a, $A D H 1 C . \mathrm{F}_{2,12}=86, \mathrm{P}<0.001$ ], [Fig. 2b, $A D H 1 A: \mathrm{F}_{2,12}=40, \mathrm{P}<0.001$ ], [Fig. $2 \mathrm{~b}, A D H 1 B: \mathrm{F}_{2,12}=313, \mathrm{P}<0.001$ ], [Fig. 2b, $A D H 1 C . \mathrm{F}_{2,12}=17.5,12 \mathrm{~h}: \mathrm{P}=0.025$, 24h: $\mathrm{P}<0.001$ ] (Fig. 2a, b). PD98059 also upregulated $A D H 1 A$, $A D H 1 B$ and $A D H 1 C$ mRNA expression [ADH1A: $\left.\mathrm{F}_{2,12}=113, \mathrm{P}<0.001\right]$, [ADH1B: $\mathrm{F}_{2,12}=24.8,20 \mathrm{mM}$ : $\mathrm{P}=0.0016,40 \mathrm{mM}: \mathrm{P}<0.001]$, [ADH1C. $\mathrm{F}_{2,12}=323, \mathrm{P}<0.001$ ] (Fig. 2c). To further confirm the effects of ERK1/2 inhibition on ADH1 transcription, we constructed ADH1A, ADH1B and ADH1C promoters and determined the effects of PD98059 on their activities. As shown in Fig. 2d, ADH1A, ADH1B and ADH1C promoter activities were all notably increased by PD98059 [ADH1A: $F_{3,16}=50.6, P<0.001$ ], [ADH1B: $F_{3}$, 16 =35, 1 mM: $P=0.0011,5$ mM: $P<0.001,20$ mM: $P<0.001$ ], [ADH1C: $\left.F_{3,16}=71.5, P<0.001\right]$. Taken together, above results indicate MEK1/2 inhibitors promote $A D H 1$ protein expression by inducing transcription activity.

\section{MEK1/2 inhibitors activate ADH1 expression in mice}

Before investigated the effects of MEK1/2 inhibitors in vivo, we initially treated BNL cells with $\mathrm{U} 0126$ and PD98059 at different concentrations. Similar to the results in HepG2 cells, MEK1/2 inhibitors increased $A D H 1$ protein expression in BNL cells [PD98059: $\left.F_{2,6}=179, P<0.001\right]$, [U0126: $F_{3,8}=49.8,0.5 \mathrm{mM}$ : $P=0.0035,2$ mM: $P<0.001,5$ mM: $P=0.032$ ] (Fig. 3a). Fig. $3 b$ and $c$ indicate ERK1/2 inhibition also promoted ADH1 transcription in BNL cells [Fig. 3b: $F_{2,6}=25.3, P=0.0011$ ], [Fig. $3 c: F_{3,16}=173, P<0.001$ ]. These results suggest MEK1/2 inhibitors can also activate mouse $A D H 1$ expression and transcription. To further define the regulatory effects of ERK1/2 on ADH1 in vivo, we fed C57BL/6J mice a normal chow (control group) or normal chow containing U0126 (U0126 group). After treatment, mouse liver samples were used to determine protein expression of pi-ERK1/2, ERK1/2 and ADH1. As shown in Fig. 3d, associated with reduced pi-ERK1/2, U0126 significantly increased $A D H 1$ protein level $\left[t_{8}=12.35, P<0.001\right]$. These results indicate ERK1/2 inhibition can induce ADH1 expression in vivo.

\section{The activation of MEK1/2 inhibitors on ADH1 expression depends on farnesoid $X$ receptor (FXR)}

Both in vitro and in vivo experiments demonstrate MEK1/2 inhibitors can increase ADH1 expression. Then we intended to further investigate the underlying mechanism. By completing a sequence alignment analysis, we found a putative FXR response element (FXRE) sequence in ADH1A, ADH1B and ADH1C promoter respectively. The sequence of the conserved FXRE motif is RGGTCAnTGACCY (R=A/G, $\mathrm{N}=\mathrm{A} / \mathrm{C} / \mathrm{G}, \mathrm{Y}=\mathrm{C} / \mathrm{T}$ ). Several studies have demonstrated activation of $\mathrm{FXR}$ can attenuate alcohol-induced liver injury $(14,15)$. Therefore, we hypothesis MEK1/2 inhibitors may regulate ADH1 via FXR. To determine it, we initially detected protein expression of FXR in response to U0126 treatment. Indeed, both concentration and time course studies show U0126 significantly upregulated FXR protein expression, 
indicating the regulatory effect of U0126 on FXR [Fig. 4a: $F_{3,8}=50.3, P<0.001$ ], [Fig. 4b: $F_{5,12}=96.6$, $P<0.001$ ] (Fig. 4a, b). To investigate the involvement of FXR in ADH1 expression, we constructed ADH1A, ADH1B and ADH1C promoters with FXRE mutation and evaluated the effect of MEK1/2 inhibitor on activities of these promoters. Fig. 4c indicated mutation of FXRE abolished the induction effects of PD98059 on ADH1A, ADH1B and ADH1C promoters. Then we transfected cells with FXR siRNA to investigate the effects of FXR on ADH1 protein expression. Fig. 4d shows FXR siRNA significantly decreased $A D H 1$ protein expression $\left[F_{5,12}=72.3, P=0.0048\right]$. Furthermore, the induction of U0126 on $A D H 1$ protein was notably blocked by FXR siRNA. Taken together, MEK1/2 inhibitor activates ADH1 expression in an FXR-dependent manner.

\section{Discussion}

Because of low molecular weight and water solubility, ethanol can distribute from the blood into all tissues quickly after consumption (6). Ethanol is harmful to various tissues. It induces neuroinflammation and neuronal apoptosis in brain (16). Chronic ethanol consumption can stimulate hepatocyte necroptosis and accelerate development of alcohol liver disease (17). Ethanol treatment can also induce oxidative stress and impair mitochondrial biogenesis in pancreatic acinar cells (18). Therefore, increasing the elimination rate of ethanol is important for body to avoid ethanol toxicity.

$A D H$ is the rate-limiting enzyme in alcohol metabolism. Mice lacking ADH showed elevated blood alcohol concentration, plasma alanine aminotransferase, and hepatic steatosis when fed ethanol (19). Increased apoptotic bodies was also observed in pancreata of ADH deficient mice (20). Compared to wild type mice, ethanol exposure significantly increased mortality of ADH deficient mice (20). These studies indicate ADH can protect mice against ethanol toxicity. Among several ADH isozymes, ADH1 plays the most important role in alcohol elimination (6). Therefore, induction of $A D H 1$ may accelerate alcohol metabolism and function as a therapeutic strategy for alcohol-related diseases.

Li et al have shown ERK1/2 is involved in alcohol liver disease (10). They found ethanol induced NF-KBinducing kinase (NIK) expression. Activated NIK recruited ERK1/2 and formed a complex to suppress PPARa signaling. As a result, liver lipid accumulation increased. This paper indicates ERK1/2 aggravated alcohol-induced liver steatosis by inhibiting PPARa-induced lipid oxidation. However, whether blockage of ERK1/2 can protect liver from alcohol toxicity by promoting ethanol metabolism remains unclear.

In current study, we determined MEK1/2 inhibitors induced ADH1 expression in human and murine hepatic cell lines by promoting its transcription. In mice, administration of mice with U0126 increased ADH1 expression in liver. Mechanistically, we found FXR siRNA and FXRE mutation in ADH1 promoters abolished MEK1/2 inhibitors-induced ADH1 expression, indicating MEK1/2 inhibitors activated ADH1 mRNA and protein expression in an FXR dependent manner. Taken together, MEK1/2 inhibitors may be potential drugs for alcohol-related diseases treatment.

\section{Declarations}




\section{Author contributions}

Xiaolin Wang conducted experiments; Yanfang Yang performed data analysis; Xiaoju Li, Yuanli Chen, Yajun Duan, Jihong Han and Xiaoxiao Yang designed experiments and wrote the manuscript.

\section{Compliance with Ethical Standards}

\section{Funding}

This work was supported by the National Natural Science Foundation of China (NSFC) Grants 81803517 to Xiaoxiao Yang, 81973316 to Jihong Han, and 31770863 to Yuanli Chen; and the China Postdoctoral Science Foundation Grants 2020M681914 to Xiaoxiao Yang.

\section{Ethical approval}

All animal procedures in this study were approved by the Ethics Committee of Nankai University and conforms to the Guide for the Care and Use of Laboratory Animals published by $\mathrm{NIH}$.

\section{Conflict of interest}

The authors have no conflicts of interest to declare that are relevant to the content of this article.

\section{References}

1. Pitel AL, Beaunieux H, Witkowski T, Vabret F, Guillery-Girard B, Quinette P et al (2007) Genuine episodic memory deficits and executive dysfunctions in alcoholic subjects early in abstinence. Alcohol Clin Exp Res 31(7):1169-1178

2. Romero-Gomez M, Grande L, Nogales MC, Fernandez M, Chavez M, Castro M (2001) Intrahepatic hepatitis $C$ virus replication is increased in patients with regular alcohol consumption. Dig Liver Dis 33(8):698-702

3. Boffetta P, Hashibe M (2006) Alcohol and cancer. Lancet Oncol 7(2):149-156

4. Stickel F, Datz C, Hampe J, Bataller R (2017) Pathophysiology and management of alcoholic liver disease: update 2016. Gut Liver 11(2):173-188

5. Rehm J, Samokhvalov AV, Shield KD (2013) Global burden of alcoholic liver diseases. J Hepatol 59(1):160-168

6. Cederbaum Al (2012) Alcohol metabolism. Clin Liver Dis 16(4):667-685

7. Roskoski R Jr (2012) ERK1/2 MAP kinases: structure, function, and regulation. Pharmacol Res 66(2):105-143 
8. Kato H, Naiki-Ito A, Naiki T, Suzuki S, Yamashita Y, Sato S et al (2016) Connexin 32 dysfunction promotes ethanol-related hepatocarcinogenesis via activation of Dusp1-Erk axis. Oncotarget 7(2):2009-2021

9. Liu Y, Saiyan S, Men TY, Gao HY, Wen C, Liu Y et al (2013) Hepatopoietin Cn reduces ethanol-induced hepatoxicity via sphingosine kinase 1 and sphingosine 1-phosphate receptors. J Pathol 230(4):365376

10. Li Y, Chen M, Zhou Y, Tang C, Zhang W, Zhong Y et al (2020) NIK links inflammation to hepatic steatosis by suppressing PPARa in alcoholic liver disease. Theranostics 10(8):3579-3593

11. Zhang S, Guo F, Yu M, Yang X, Yao Z, Li Q et al (2020) Reduced Nogo expression inhibits diet-induced metabolic disorders by regulating ChREBP and insulin activity. J Hepatol 73(6):1482-1495

12. Aroor AR, Restrepo RJ, Kharbanda KK, Shukla SD. Erratum to: Epigenetic histone modifications in a clinically relevant rat model of chronic ethanol-binge-mediated liver injury. Hepatol Int. 2014;8 Suppl 2:431

13. Chen CS, Wu CH, Lai YC, Lee WS, Chen HM, Chen RJ et al (2008) NF-kB-activated tissue transglutaminase is involved in ethanol-induced hepatic injury and the possible role of propolis in preventing fibrogenesis. Toxicology 246(2-3):148-157

14. Wu W, Zhu B, Peng X, Zhou M, Jia D, Gu J (2014) Activation of farnesoid X receptor attenuates hepatic injury in a murine model of alcoholic liver disease. Biochem Biophys Res Commun $443(1): 68-73$

15. Livero FA, Stolf AM, Dreifuss AA, Bastos-Pereira AL, Chicorski R, de Oliveira LG et al (2014) The FXR agonist 6ECDCA reduces hepatic steatosis and oxidative stress induced by ethanol and low-protein diet in mice. Chem Biol Interact 217:19-27

16. Sangaunchom P, Dharmasaroja P (2020) Caffeine potentiates ethanol-induced neurotoxicity through mTOR/p70S6K/4E-BP1 inhibition in SH-SY5Y cells. Int J Toxicol 39(2):131-140

17. Ji C, Nagaoka K, Zou J, Casulli S, Lu S, Cao KY et al (2019) Chronic ethanol-mediated hepatocyte apoptosis links to decreased TET1 and 5-hydroxymethylcytosine formation. FASEB J 33(2):18241835

18. Srinivasan MP, Bhopale KK, Caracheo AA, Amer SM, Khan S, Kaphalia L et al (2020) Activation of AMP-activated protein kinase attenuates ethanol-induced ER/oxidative stress and lipid phenotype in human pancreatic acinar cells. Biochem Pharmacol 180:114174

19. Srinivasan MP, Bhopale KK, Amer SM, Wan J, Kaphalia L, Ansari GS et al. Linking dysregulated AMPK signaling and ER stress in ethanol-induced liver injury in hepatic alcohol dehydrogenase deficient deer mice. Biomolecules. 2019;9(10)

20. Bhopale KK, Wu H, Boor PJ, Popov VL, Ansari GA, Kaphalia BS (2006) Metabolic basis of ethanolinduced hepatic and pancreatic injury in hepatic alcohol dehydrogenase deficient deer mice. Alcohol 39(3):179-188

\section{Figures}


a
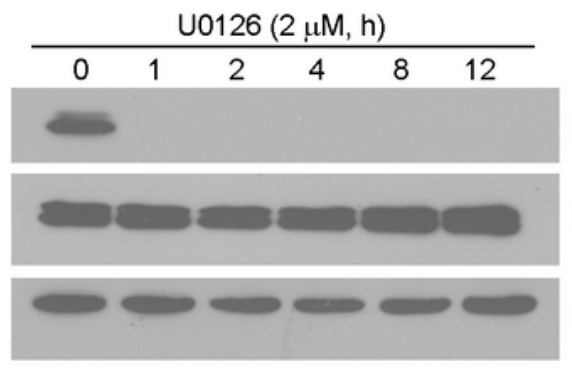

$\mathrm{pi-ERK1/2}$

ERK1/2

GAPDH

C

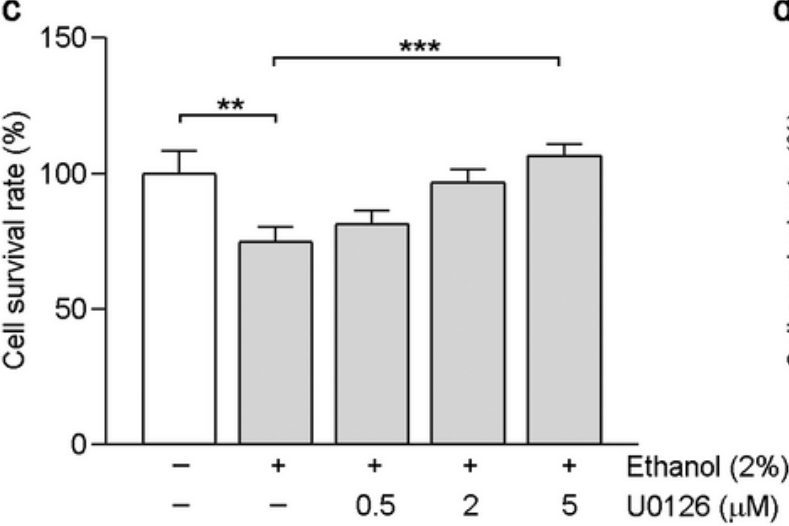

e
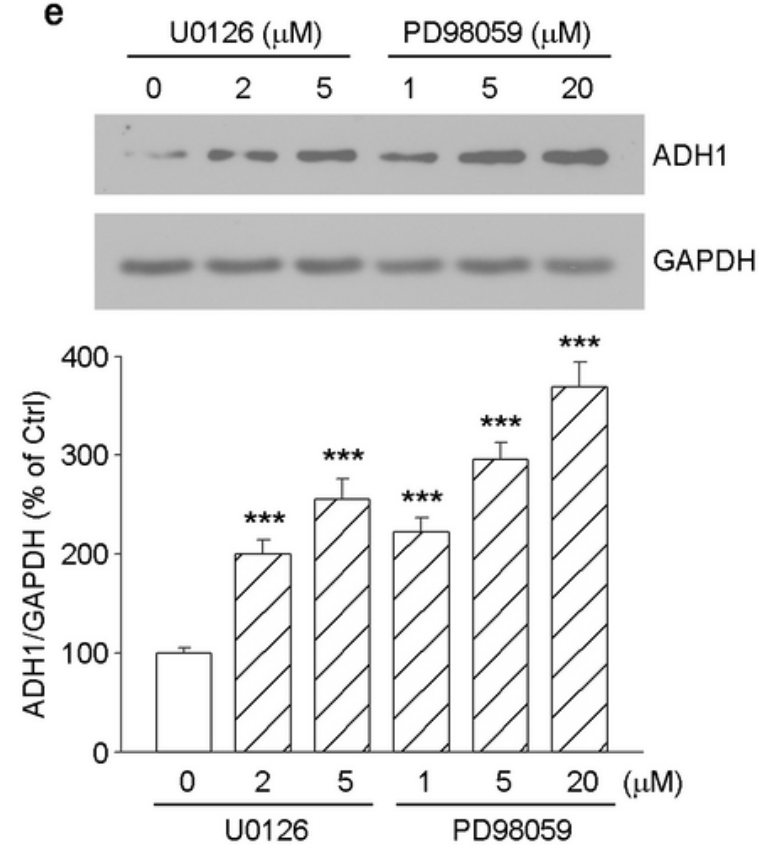

b

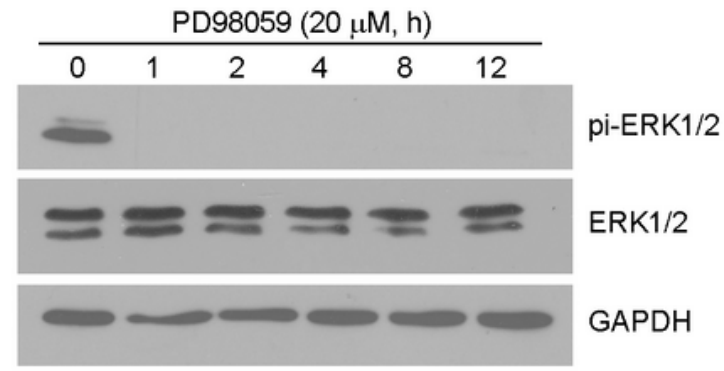

d

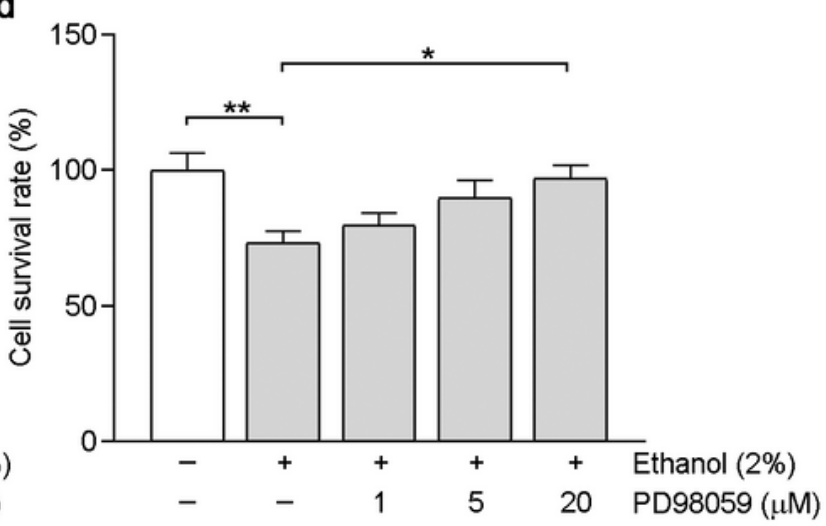

f U0126 (5 $\mu \mathrm{M}, \mathrm{h})$
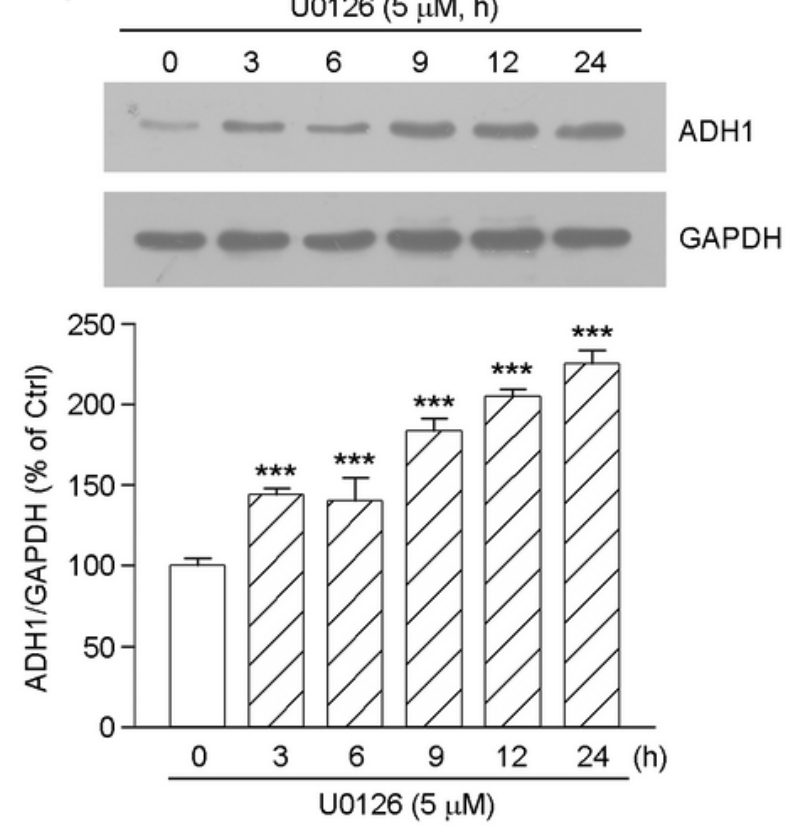

\section{Figure 1}

MEK1/2 inhibitors induce ADH1 expression in HepG2 cells a, b: HepG2 cells were treated with U0126 (a) and PD98059 (b) for the indicated time followed by total protein extraction and determination of phosphorylated ERK1/2 (pi-ERK1/2) and ERK1/2 by Western blot ( $n=3) ; c$, d: HepG2 cells were treated with $2 \%$ ethanol (V/V) plus U0126 (c) or PD98059 (d) at the indicated concentrations overnight followed by determination of cell survival rate using the MTT assay kit ( $n=5)$; e, f: HepG2 cells were treated with 
U0126 or PD98059 at the indicated concentrations for $16 \mathrm{~h}$ (e) or with $5 \square \mathrm{M} \mathrm{U0126}$ for the indicated time (f). Total cellular protein was used to determine the expression of $A D H 1$ by Western blot with quantitative analysis $(n=3) .{ }^{*}<<0.05,{ }^{*} P<0.01,{ }^{*} * P<0.001$ vs. control or as indicated.

a
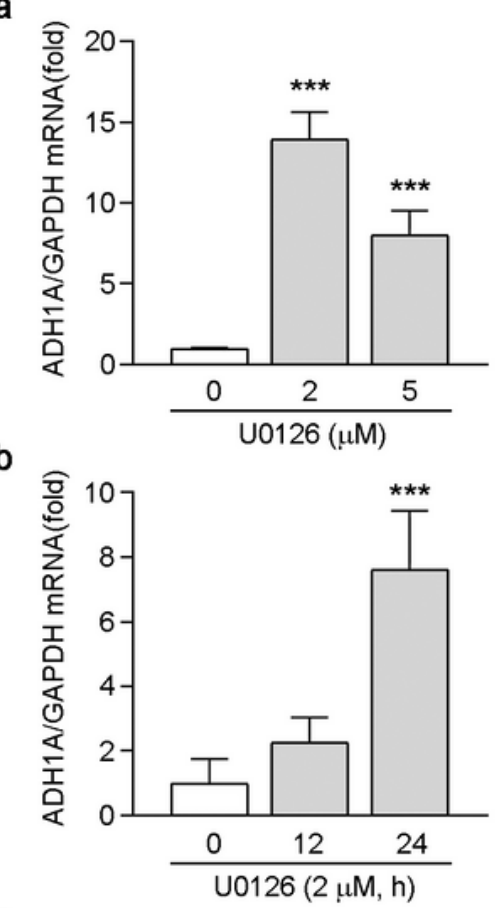

C

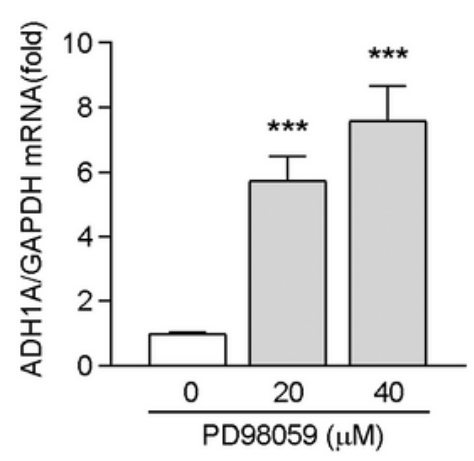

d

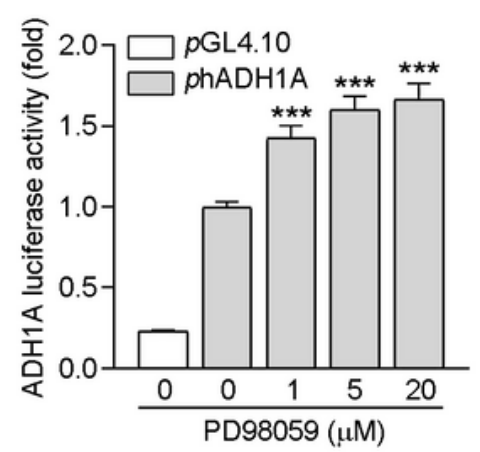

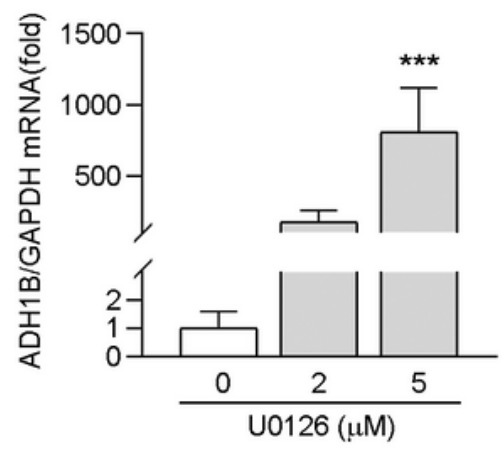
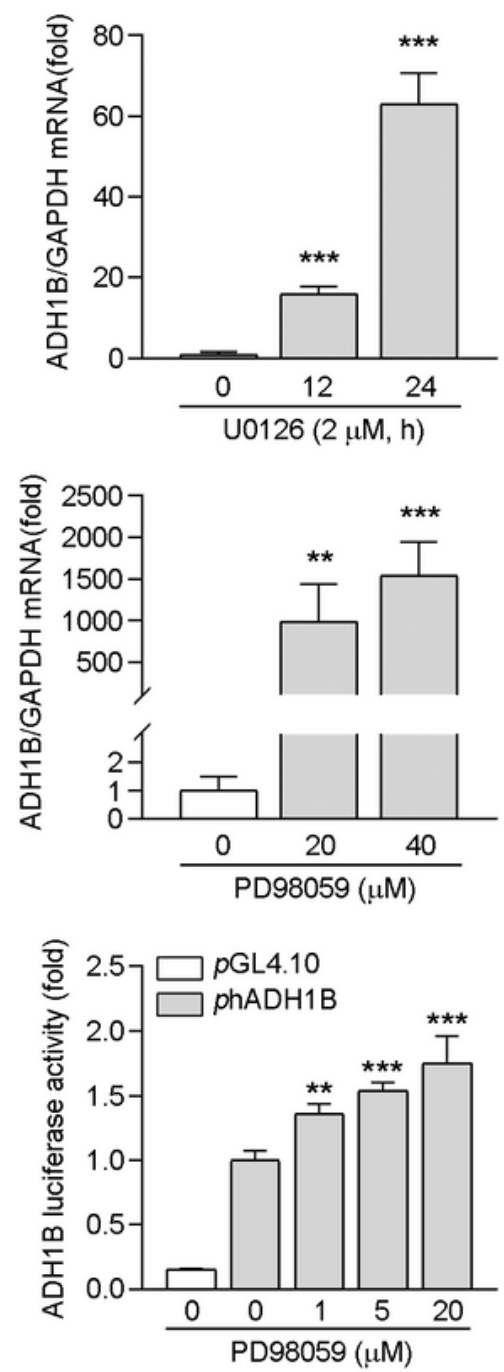
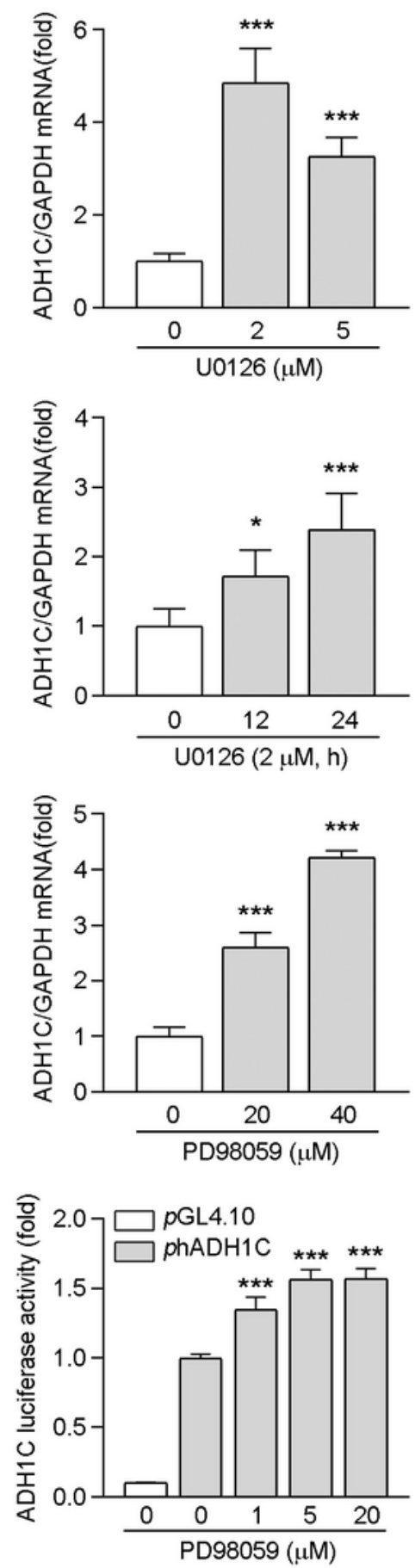

Figure 2

MEK inhibitors enhance AHD1 transcription in HepG2 cells a-c: HepG2 cells received treatment of U0126 (a) or PD98059 (c) at the indicated concentrations for $16 \mathrm{~h}$ or 2 GM U0126 for the indicated time (b). 
Expression of ADH1A, ADH1B and ADH1C mRNA were determined by qRT-PCR; d: HepG2 cells were transfected with pGL4.10 vector or human ADH1A, ADH1B, ADH1C promoters and Renilla luciferase. After treatment with PD98059 at the indicated concentrations for $16 \mathrm{~h}$, promoter activity of ADH1A, ADH1B and ADH1C were determined using dual-luciferase assay kit. ${ }^{*} P<0.01, * \star * P<0.001$ vs. control $(n=5)$.

a

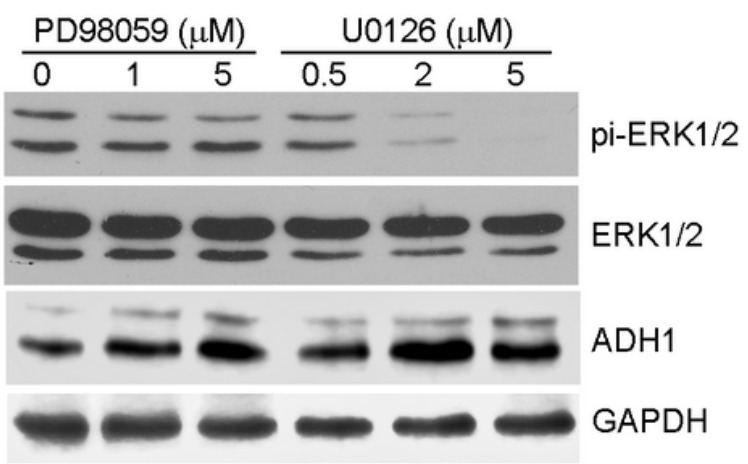

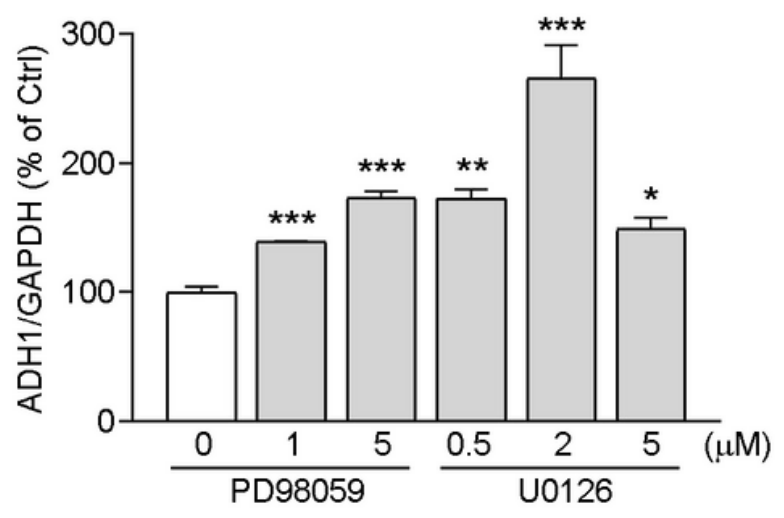

d Control U0126

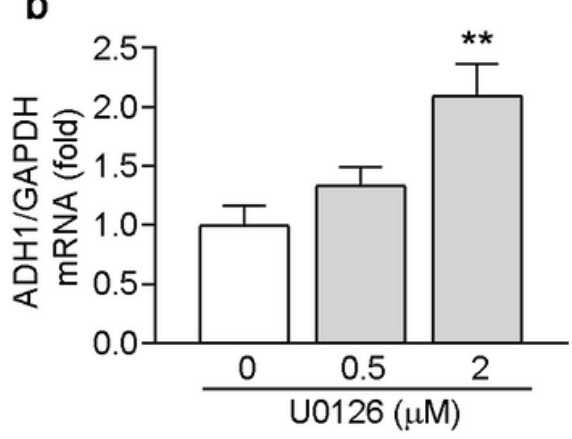

C

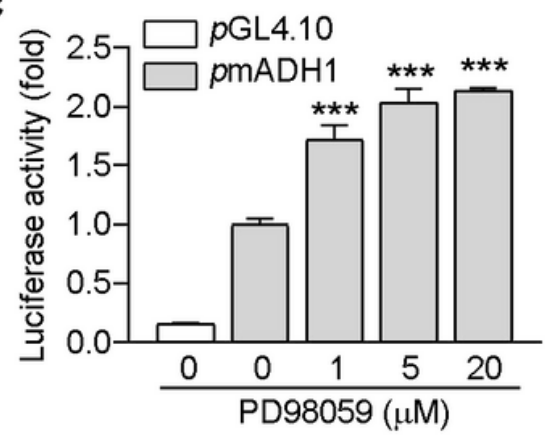

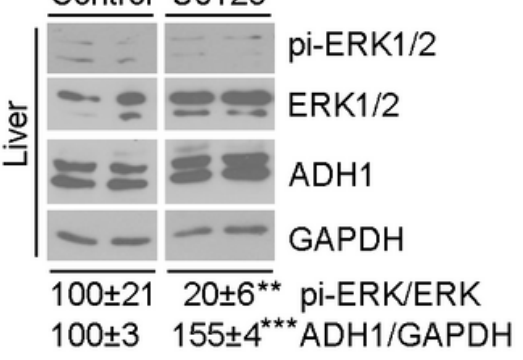

\section{Figure 3}

MEK1/2 inhibitors activate ADH1 expression in BNL cells and C57BL/6 mice a-b: BNL cells were treated with PD98059 or U0126 at the indicated concentrations for $16 \mathrm{~h}$. Protein expression of pi-ERK1/2, ERK1/2 and ADH1 (a) were determined by Western blot with quantitative analysis of ADH1 band density; mRNA expression of ADH1 (b) was determined by qRT-PCR ( $n=3)$. c: BNL cells were transfected with pGL4.10 vector or mouse ADH1 promoter and Renilla luciferase. After treatment with PD98059 at the indicated concentrations for $16 \mathrm{~h}$, promoter activity of $\mathrm{ADH} 1$ was determined using dual-luciferase assay kit $(\mathrm{n}=5)$. d: Male C57BL/6J mice ( 6-week old) were fed normal chow (control) or normal chow containing U0126 (1.5 mg/day/kg bodyweight, U0126 group) for five weeks. After treatment, liver samples were collected and used to determine protein expression of pi-ERK1/2, ERK1/2 and ADH1 by Western blot with

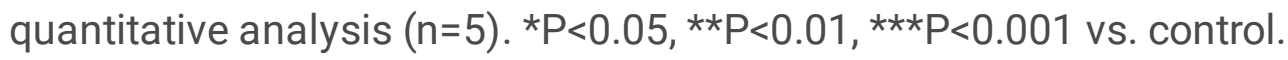


a
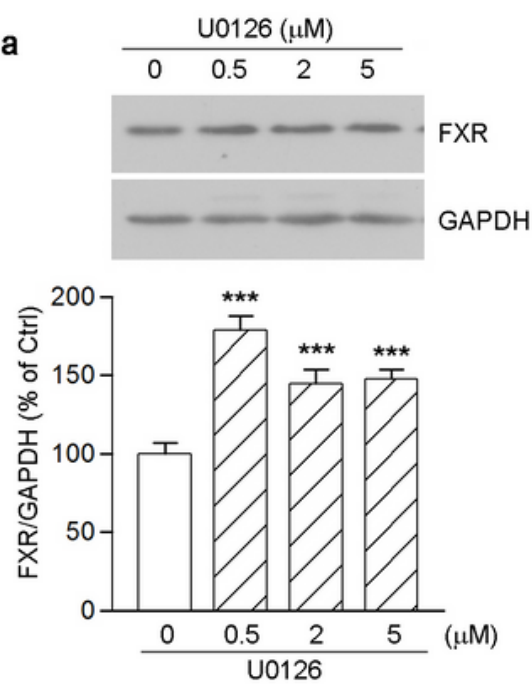

C

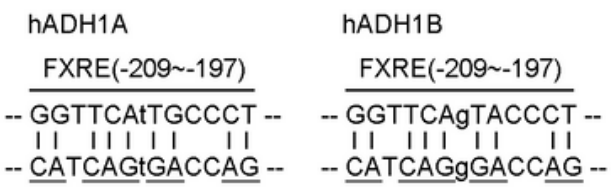

b
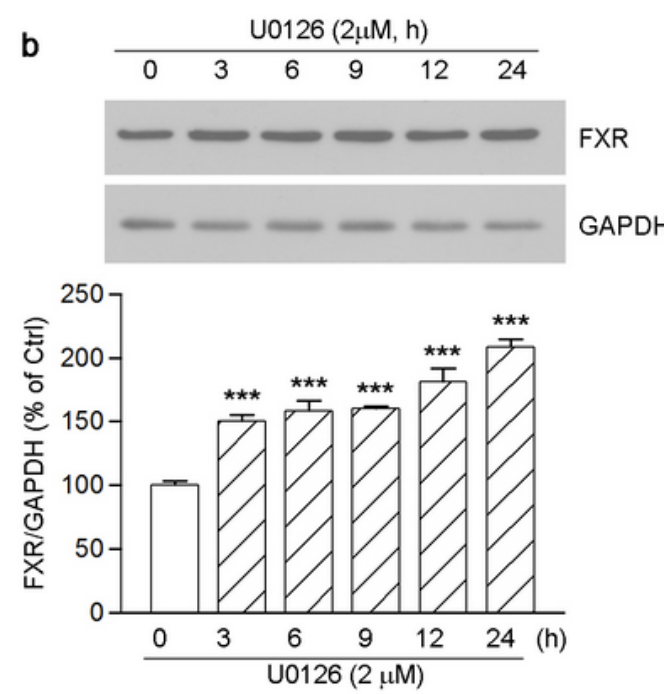

hADH1C

FXRE(-141 -129)

-- GGTTCGgTGCCCA -- Sequence of normal FXRE

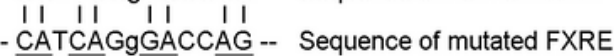
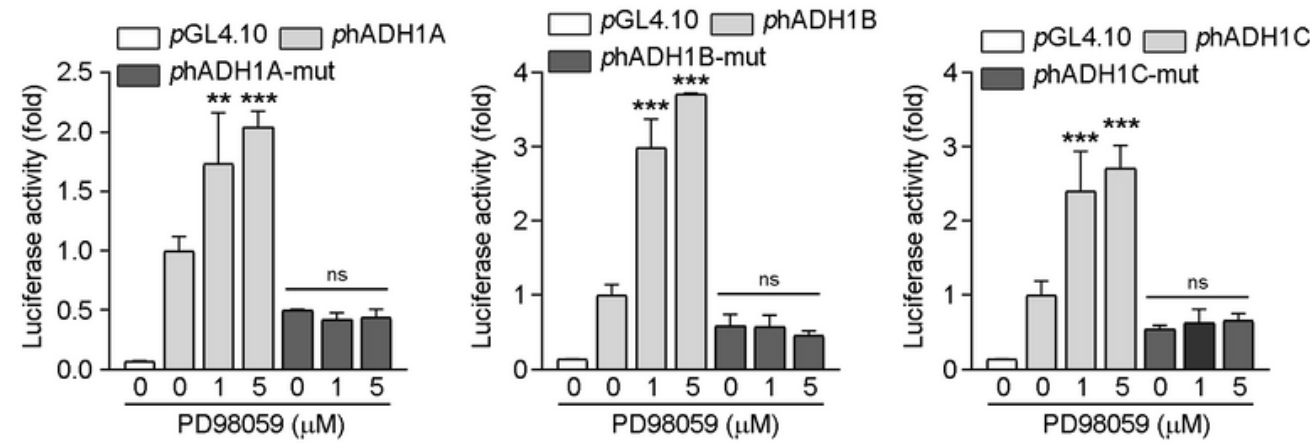

d

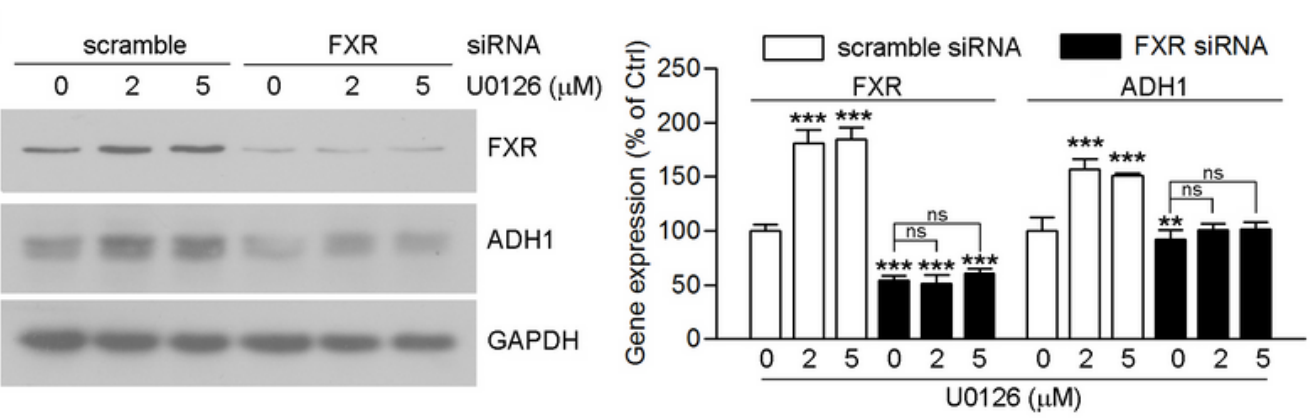

\section{Figure 4}

U0126 induces ADH1 expression in an FXR dependent manner a, b: HepG2 cells received treatment of U0126 (a) at the indicated concentrations for $16 \mathrm{~h}$ or $2 \mathrm{QM} \mathrm{U} 0126$ for the indicated time (b). Protein expression of FXR was determined by Western blot with quantitative analysis $(n=3)$. c: HepG2 cells were transfected with pGL4.10 vector or human ADH1A, ADH1B, ADH1C promoters with or without FXRE mutation and Renilla luciferase. After treatment with PD98059 at the indicated concentrations for $16 \mathrm{~h}$, 
promoter activities were determined using dual-luciferase assay kit $(n=5)$. The sequences of human $\mathrm{ADH} 1 \mathrm{~A}, \mathrm{ADH} 1 \mathrm{~B}$ and $\mathrm{ADH} 1 \mathrm{C}$ or mutated promoters were listed (the mutated nucleotides in each FXRE were underlined). d: HepG2 cells were transfected with scramble siRNA or FXR siRNA for $24 \mathrm{~h}$, followed by treatment with $\mathrm{U} 0126$ at the indicated concentrations for $16 \mathrm{~h}$. Protein expression of FXR and ADH1 were determined by Western blot with quantitative analysis $(n=3) .{ }^{*} P<0.01, * * * P<0.001$ vs. control; $n$, not significantly different.

\section{Supplementary Files}

This is a list of supplementary files associated with this preprint. Click to download.

- supplementarymaterials.pdf 